\title{
Synthesis of $\gamma-\mathrm{Al}_{2} \mathrm{O}_{3}$ nanowires through a boehmite precursor route
}

\author{
QI YANG \\ School of Materials Engineering, Shanghai University of Engineering Science, Shanghai 201620, China
}

MS received 3 May 2008; revised 22 January 2011

\begin{abstract}
Crystalline $\gamma-\mathrm{Al}_{2} \mathrm{O}_{3}$ nanowires with diameter, $20-40 \mathrm{~nm}$, length above $600 \mathrm{~nm}$ and aspect ratio above 30 have been successfully synthesized by thermal decomposition of boehmite $(\gamma$-AlOOH) precursors obtained via hydrothermal route by using $\mathrm{AlCl}_{3}, \mathrm{NaOH}$ and $\mathrm{NH}_{3}$ as starting materials. Thermogravimetric analysis (TG), differential thermal analysis (DTA), X-ray diffraction (XRD), transmission electron microscope (TEM), selected area electron diffraction (SAED) and high resolution transmission electron microscope (HRTEM) were used to characterize the features of the as-made $\gamma-\mathrm{Al}_{2} \mathrm{O}_{3}$ nanowires and their $\gamma$-AlOOH precursors. The $\mathrm{pH}$ value of the solution and the mixed precipitant play important roles in the formation of $\gamma$-AlOOH nanowires. After calcination at $5_{00}^{\circ} \mathrm{C}$ for $2 \mathrm{~h}$, the orthorhombic $\gamma$-AlOOH transforms to cubic $\gamma-\mathrm{Al}_{2} \mathrm{O}_{3}$ and retains nanowire morphology.
\end{abstract}

Keywords. $\quad \gamma-\mathrm{Al}_{2} \mathrm{O}_{3} ; \gamma$-AlOOH; nanowires; hydrothermal route; $\mathrm{pH}$ value.

\section{Introduction}

Since the discovery of carbon nanotubes (Iijima 1991), materials with one-dimensional (1D) nanostructures have received continuous attention because of their uniquely electronic, mechanical and chemical properties and their use as building blocks for nanodevices (Pan et al 2001; Baughman et al 2002). Many methods have been developed for the preparation of the 1D nanostructures, including vapour-liquid-solid (VLS) methods (Hu et al 1999), chemical vapour deposition (CVD) (Rao et al 2003), thermal evaporation (Kong et al 1998), template assisted approaches (Han et al 1997), and solution-phase methods (Jun et al 2006; Wang and Li 2006). Among the various approaches, the solution-phase method seems to be low-cost and efficient for large-scale production (Xia et al 1999).

As a type of important structural ceramic material, alumina has applications in absorbent, catalyst carrier and reinforcement of ceramic composites for its high strength, corrosion resistance, chemical stableness, low thermal conductivity, and good electrical insulation (Hellmig and Ferkel 1999; Peng et al 2002). The synthesis of nanostructured alumina, especially one-dimensional nanostructures, has received considerable interest due to its novel properties, such as high elastic modulus, thermal and chemical stability, and optical characteristics (Moc et al 1995). Up to date, the most common synthetic strategies of crystalline alumina nanowires are based on vapour-based synthetic methods. Single-crystal $\mathrm{Al}_{2} \mathrm{O}_{3}$ fibres were first produced at Lawrence Berkeley laboratory, using a basal sapphire (single crystal) substrate. Zhou

(qiiyang@163.com) et al (2002) synthesized crystalline alumina nanowires with a diameter of about $50 \mathrm{~nm}$ and length of about $2 \mu \mathrm{m}$ in a catalyst-assisted process using iron as catalyst. Tang et al (2001) grew $\mathrm{Al}_{2} \mathrm{O}_{3}$ nanowires by heating a mixture of $\mathrm{Al}$, $\mathrm{SiO}_{2}$ and $\mathrm{Fe}_{2} \mathrm{O}_{3}$ catalyst.

Recently, considerable efforts have been focused on the preparation of boehmite $(\gamma-\mathrm{AlOOH})$ nanostructures by solution-phase routes because they are usually used as the precursors for the synthesis of alumina nanostructures. Chen et al (2007) present a hydrothermal route to synthesize $1 \mathrm{D}$ and 2D $\gamma$-AlOOH nanomaterials under either acidic or basic conditions by using aluminum nitrate, ethylenediamine or hexamethylenetetramine as starting materials. Under hydrothermal conditions, they prepared $\gamma-\mathrm{AlOOH}$ with two distinct 1D straw-bundle-like and 2D plate-like morphology by manipulating the acidity of the reaction solution at $\mathrm{pH}=5$ and 10 , respectively (Chen et al 2007). $\gamma$ alumina single-crystalline nanorods with a diameter of about 20-50 $\mathrm{nm}$ and length of about 200-300 $\mathrm{nm}$ were synthesized by thermal decomposition of boehmite precursor which was prepared by solvothermally treating $\mathrm{AlCl}_{3} \cdot 6 \mathrm{H}_{2} \mathrm{O}, \mathrm{NaOH}$, sodium dodecyl benzene sulfonate in water and dimethylbenzene mixed solvents (Ma et al 2007). In this paper, we report a simple hydrothermal route to the synthesis of $\gamma-\mathrm{AlOOH}$ nanowires with much longer length and larger aspect ratio by using $\mathrm{AlCl}_{3}, \mathrm{NaOH}$ and $\mathrm{NH}_{3} \cdot \mathrm{H}_{2} \mathrm{O}$ as starting materials. The $\gamma$-AlOOH nanowires were used as precursors for the synthesis of $\gamma-\mathrm{Al}_{2} \mathrm{O}_{3}$ nanowires by thermal decomposition.

\section{Experimental}

All chemicals were of analytical grade reagents and used as received without further purification. All experiments were 
conducted under air atmosphere. In a typical synthesis experiment, $\mathrm{AlCl}_{3} \cdot 6 \mathrm{H}_{2} \mathrm{O}(12 \mathrm{~g})$ was dissolved in $50 \mathrm{ml}$ distilled water under vigorous magnetic stirring at room temperature to form $\mathrm{AlCl}_{3}$ solution $(1.00 \mathrm{M}) .25 \mathrm{ml} \mathrm{NH} \mathrm{NH}_{3} \cdot \mathrm{H}_{2} \mathrm{O}$ solution $(1.00 \mathrm{M})$ was added into $25 \mathrm{ml} \mathrm{NaOH}$ solution $(1.00 \mathrm{M})$ to prepare mixed precipitant. Subsequently, the mixed precipitant was added drop by drop to the $\mathrm{AlCl}_{3}$ solution to give lacteous precipitates immediately. At this point, the $\mathrm{pH}$ value of the reaction mixture was 5 . The resulting reaction mixture was transferred into a $100 \mathrm{ml}$ Teflon-lined autoclave, which was then sealed and kept in an electric oven at $200^{\circ} \mathrm{C}$. After $24 \mathrm{~h}$, the autoclave was air-cooled to room temperature. The resultant colloidal product was separated from the solution by centrifugation, washed with water and ethanol several times and dried at $60^{\circ} \mathrm{C}$ in vacuum for $12 \mathrm{~h}$. Finally, the white powder (boehmite) was put into an alumina crucible in a tube furnace and heated to $500^{\circ} \mathrm{C}$ in air with a heating rate of $3^{\circ} \mathrm{C} / \mathrm{min}$ and kept at $500^{\circ} \mathrm{C}$ for $2 \mathrm{~h}$.

The X-ray diffraction (XRD) patterns of the obtained products were recorded on a BRUKER-AXS X-ray powder diffractometer with a graphite monochromator and $\mathrm{CuK} \alpha$ radiation $(\lambda=0.154178 \mathrm{~nm})$ at $40 \mathrm{kV}$ and $60 \mathrm{~mA}$ in a $2 \theta$ range from $10-70^{\circ} \mathrm{C}$ at room temperature. Transmission electron microscopy (TEM) images, selected-area electron diffraction (SAED) patterns and high resolution transmission electron microscopy (HRTEM) images were taken with a JEOL-2010 transmission electron microscope. TG and DTA were performed on a TG 2050 thermogravimetric analysis (TG) from room temperature to $800^{\circ} \mathrm{C}$ and a DTA 1600 differential thermal analysis (DTA) from room temperature to $1200^{\circ} \mathrm{C}$ at a heating rate of $10^{\circ} \mathrm{C} / \mathrm{min}$ in air.

\section{Results and discussion}

Figure 1 shows XRD patterns of the obtained products. All of the diffraction peaks in figure 1(a) can be indexed to an orthorhombic phase of $\gamma-\mathrm{AlOOH}$, and the cell parameters, $a=0.5293 \mathrm{~nm}, b=0.5264 \mathrm{~nm}$ and $c=0.5318 \mathrm{~nm}$, are compatible with the literature values of JCPDS No. 21-1307. Similarly, all of the diffraction peaks in figure 1(b) can be indexed to a cubic phase of $\gamma-\mathrm{Al}_{2} \mathrm{O}_{3}$, and the lattice constants, $a=0.9739 \mathrm{~nm}$ and $c=0.2876 \mathrm{~nm}$ are also consistent with the values of JCPDS No. 10-0425. Therefore, the XRD analysis results indicate that phase-pure $\gamma$-AlOOH was synthesized via a hydrothermal method with $\mathrm{AlCl}_{3}, \mathrm{NaOH}$ and $\mathrm{NH}_{3} \cdot \mathrm{H}_{2} \mathrm{O}$ as starting materials at $200^{\circ} \mathrm{C}$ for $24 \mathrm{~h}$, and the $\gamma$-AlOOH was completely converted to $\gamma-\mathrm{Al}_{2} \mathrm{O}_{3}$ after heat treatment in air at $500^{\circ} \mathrm{C}$ for $2 \mathrm{~h}$. The intensity of the (400) peak in figure 1(b) is stronger than the other peaks, indicating that the (400) planes may be the preferential growth direction of $\gamma-\mathrm{Al}_{2} \mathrm{O}_{3}$.

The morphology and structure of the precursor $\gamma$-AlOOH and $\gamma-\mathrm{Al}_{2} \mathrm{O}_{3}$ were investigated with TEM, SAED and HRTEM. Figure 2(a) shows the TEM image of the $\gamma$ $\mathrm{AlOOH}$. It is clear that $\gamma-\mathrm{AlOOH}$ comprises of a large

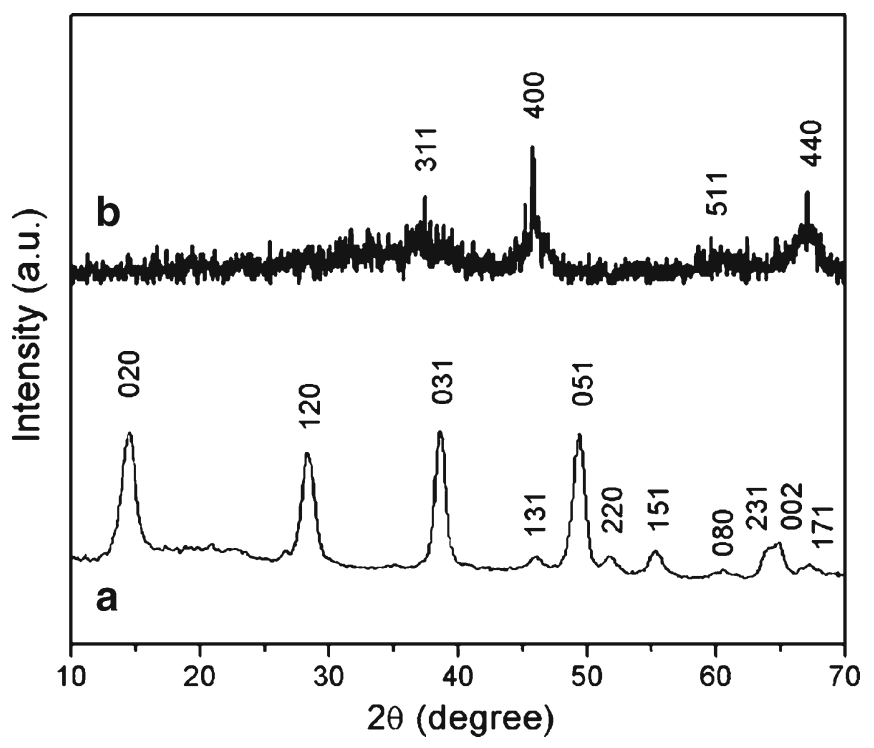

Figure 1. XRD patterns of $\mathbf{a}$. the precursor without calcination and $\mathbf{b}$. the precursor calcinated at $500^{\circ} \mathrm{C}$ for $2 \mathrm{~h}$.

quantity of nanowires with diameters in the range 20$40 \mathrm{~nm}$, lengths above $600 \mathrm{~nm}$ and aspect ratio above 30 . The HRTEM image of a single nanowire in figure 2(b) illustrates a lattice fringe of $\sim 0.32 \mathrm{~nm}$, corresponding to the separation between the neighbouring (120) lattices. The symmetrically scattered spots of the inset in figure 2(b) clearly show the single-crystalline of the as-synthesized $\gamma$-AlOOH nanowires (inset of figure 2(b)). Figure 2(c) displays TEM micrograph of $\gamma-\mathrm{Al}_{2} \mathrm{O}_{3}$ nanowires prepared by the thermal decomposition of the $\gamma$-AlOOH nanowires. The size and morphology of $\gamma-\mathrm{Al}_{2} \mathrm{O}_{3}$ are similar to those of the $\gamma$-AlOOH precursor. It can be concluded that during the conversion from $\gamma$ AlOOH to $\gamma-\mathrm{Al}_{2} \mathrm{O}_{3}$, morphology of the products is reserved on the whole. HRTEM was also used to analyse the lattice structures (figure $2(\mathrm{~d})$ ) of the $\gamma-\mathrm{Al}_{2} \mathrm{O}_{3}$ nanowires, and the selected-area electron diffraction pattern (inset of figure 2(d)) was also recorded. The HRTEM image clearly shows that the lattice fringe is $\sim 0.2 \mathrm{~nm}$, consistent with that of the (400) plane of $\gamma-\mathrm{AlOOH}$ crystal, and the preferential growth direction is (400). The inset of figure 2(d) presents the selected area electron diffraction (SAED) pattern taken from a single nanowire, which can be indexed as single-crystalline cubic structural $\gamma-\mathrm{Al}_{2} \mathrm{O}_{3}$.

In order to investigate the influence of $\mathrm{pH}$ value and the types of precipitant on the formation of the $\gamma-\mathrm{AlOOH}$ nanowires, different experiments were carried out by changing the $\mathrm{pH}$ value or using single precipitant such as $\mathrm{NaOH}$ or $\mathrm{NH}_{3} \cdot \mathrm{H}_{2} \mathrm{O}$. Figure 3(a) shows the TEM images of the $\gamma$ $\mathrm{AlOOH}$ prepared at $\mathrm{pH}=4$, indicating that they have onedimension morphology and agglomerate with each other, the surface of one dimension $\gamma$-AlOOH exhibits zigzag patterns. The SAED pattern with rings consisting of spots illustrates the polycrystalline nature of the sample (inset of figure 3(a)). 

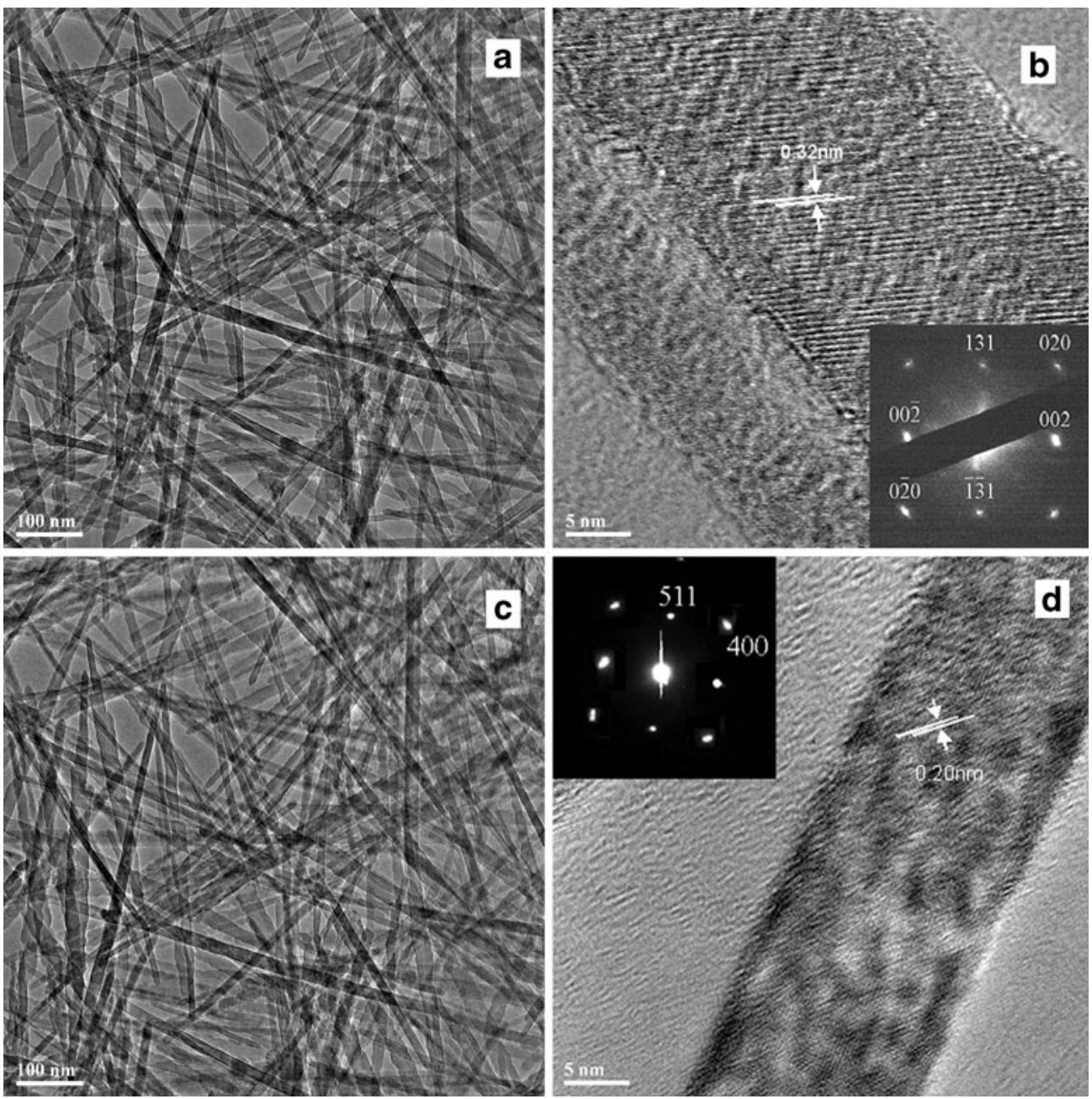

Figure 2. a. TEM image of the $\gamma$-AlOOH nanowires; $\mathbf{b}$. HRTEM image of the $\gamma$-AlOOH nanowires and SAED pattern (inset); $\mathbf{c}$. TEM image of the $\gamma-\mathrm{Al}_{2} \mathrm{O}_{3}$ nanowires and $\mathbf{d}$. HRTEM image of $\gamma-\mathrm{Al}_{2} \mathrm{O}_{3}$ nanowires and SAED pattern (inset).

When the $\mathrm{pH}$ value increased to 6 , the $\gamma$-AlOOH nanorods with shorter length and bigger diameter were obtained (figure 3(b)). Figure 3(c) shows TEM image of the sample prepared under basic conditions $(\mathrm{pH}=10)$, clearly demonstrating the sample to consist of many nanoflakes with a width of 50-100 nm. In our further experiment, we also found that when the $\mathrm{pH}$ was changed to lower values, no precipitates were observed in the solution after hydrothermal reaction.

The types of precipitant also play important role in the formation of $\gamma$-AlOOH nanowires. Figures 3(d) and (e) show the images of $\gamma$-AlOOH prepared with single precipitant of $\mathrm{NaOH}$ solution $(1 \mathrm{M})$. It can be clearly seen that the products have needle-like shape morphology with a diameter of 10-30 nm and length of 100-200 nm, and their surface exhibits zig-zag patterns. Figure 3(f) shows the image of $\gamma$-AlOOH prepared with the single precipitant of $\mathrm{NH}_{3} \cdot \mathrm{H}_{2} \mathrm{O}$ solution $(1 \mathrm{M})$. The products are not pure needlelike $\gamma$-AlOOH and contain some $\gamma$-AlOOH nanoparticles, compared with the result shown in figure 3(d).
The growth of $\gamma$-AlOOH nanowires could be attributed to the 2D framework structure of boehmite $\mathrm{AlOOH}$ containing lamellae (Chen and Lee 2007; Chen et al 2007). The formation mechanism of the $\mathrm{AlOOH}$ nanowires can be proposed as follows:

$$
\begin{aligned}
& \mathrm{AlCl}_{3}+3 \mathrm{H}_{2} \mathrm{O} \rightarrow \mathrm{Al}(\mathrm{OH})_{3} \text { (amorphous) }+3 \mathrm{HCl}, \\
& \mathrm{NaOH}+\mathrm{HCl} \rightarrow \mathrm{NaCl}+\mathrm{H}_{2} \mathrm{O}, \\
& \mathrm{NH}_{3} \cdot \mathrm{H}_{2} \mathrm{O}+\mathrm{HCl} \rightarrow \mathrm{NH}_{4} \mathrm{Cl}+\mathrm{H}_{2} \mathrm{O}, \\
& \mathrm{Al}(\mathrm{OH})_{3} \rightarrow \gamma-\mathrm{AlOOH}+\mathrm{H}_{2} \mathrm{O}(\mathrm{pH}=5) .
\end{aligned}
$$

When $\mathrm{AlCl}_{3}$ is added into water, the formed transparent solution indicates that $\mathrm{Al}(\mathrm{OH})_{3}$ can hardly be obtained in solution. By adding mixed precipitant of $\mathrm{NaOH}$ and ammonia into the transparent solution, $\mathrm{Al}(\mathrm{OH})_{3}$ with colloidal form generates immediately, because $\mathrm{NaOH}$ and ammonia remove 

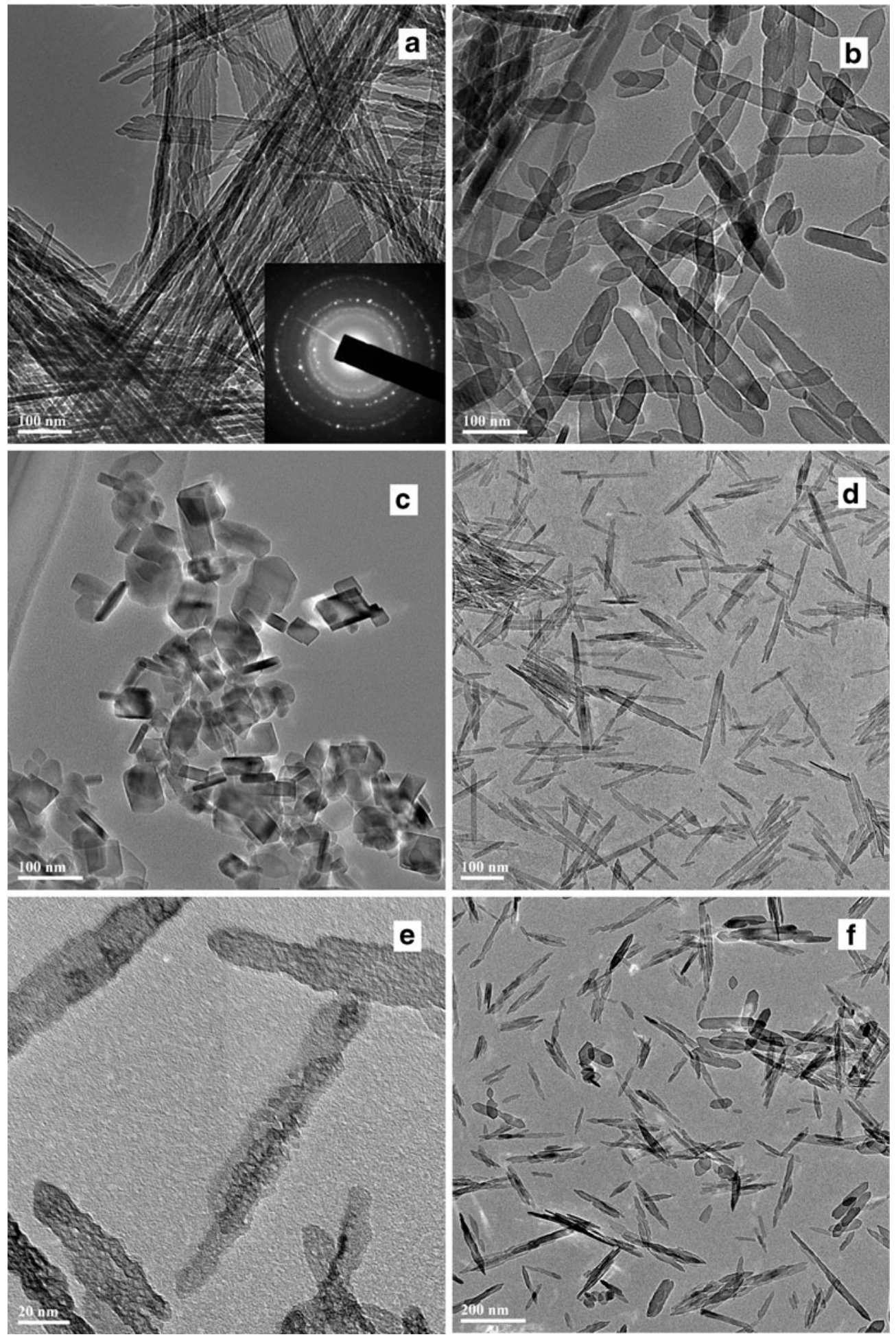

Figure 3. TEM and HRTEM images of the $\gamma$-AlOOH prepared at $200^{\circ} \mathrm{C}$ for $24 \mathrm{~h}$ : a. with mixed precipitant under $\mathrm{pH}=4$, b. with mixed precipitant under $\mathrm{pH}=5$, c. with mixed precipitant under $\mathrm{pH}=10$, d. with single precipitant of $\mathrm{NaOH}(1 \mathrm{M})$ under $\mathrm{pH}=5$, e. amplified image of figure 3d, f. with single precipitant of ammonia $(1 \mathrm{M})$ under $\mathrm{pH}=5$. 
the protons generated on the formation of $\mathrm{Al}(\mathrm{OH})_{3}$ from $\mathrm{AlCl}_{3}$ and therefore, pushes the reaction to right direction in equilibrium (1). Under the high temperature hydrothermal condition, the amorphous colloid $\mathrm{Al}(\mathrm{OH})_{3}$ will be converted into $\gamma$-AlOOH. Figure 4 illustrates the characteristic structure of the $\gamma$-AlOOH. One monolayer is constructed by octahedra with an aluminum atom near their centre, two hydroxyls and four oxygen atoms in their vertices. The layers are held together by hydrogen bonds between the $\mathrm{OH}^{-}$ groups of each octahedron. Under acidic conditions, the reaction solution contains protons that would combine with the hydroxyl oxygen-lone pairs to give aqua ligands and therefore, destroy the $\gamma$-AlOOH layers. The separated layers subsequently curl to form $1 \mathrm{D}$ nanostructures via the scrolling-growth route (Chen et al 2007).

It is generally accepted that many experimental factors could affect the morphology and size of the final products. In our experiment, the $\mathrm{pH}$ value of the solution and the mixed precipitation of $\mathrm{NaOH}$ and ammonia play important roles in the formation of $\gamma$-AlOOH nanowires. However, currently the detailed growth mechanism is still not well understood, but it is believed that the $\gamma-\mathrm{AlOOH}$ nanowires are significantly sensitive to $\mathrm{pH}$ value and starting material. Further investigation is underway and the detailed discussions will appear in the future.

The thermal stability of the $\gamma-\mathrm{AlOOH}$ nanowires was studied by thermal analysis in air atmosphere. Figure 5 shows the TG and DTA curves of as-made $\gamma$-AlOOH nanowires. It is found that three distinct steps of weight loss are discernible, in the range of (i) $t<200^{\circ} \mathrm{C}$, (ii) $200-500^{\circ} \mathrm{C}$ and (iii) $500-800^{\circ} \mathrm{C}$. The first step with a weight loss of $1 \%$ corresponds to the desorption of dissociated water, and the second step with $15 \%$ loss can be attributed to the partial dehydroxylation occurring when boehmite transformed into $\gamma-\mathrm{Al}_{2} \mathrm{O}_{3}$. While the temperature increased from $500-800^{\circ} \mathrm{C}$, the weight loss of $2 \%$ was associated with further elimination of the residual hydroxyls in the crystalline structure of the $\gamma-\mathrm{Al}_{2} \mathrm{O}_{3}$ (KnoK zinger and Ratnasamy 1978; Liu and

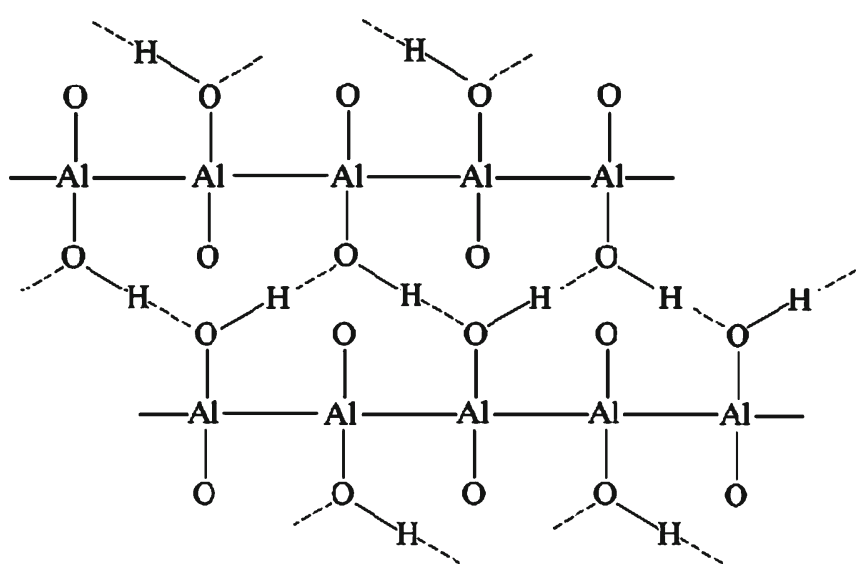

Figure 4. Schematic diagrams of $\gamma$-AlOOH lamellar structure.

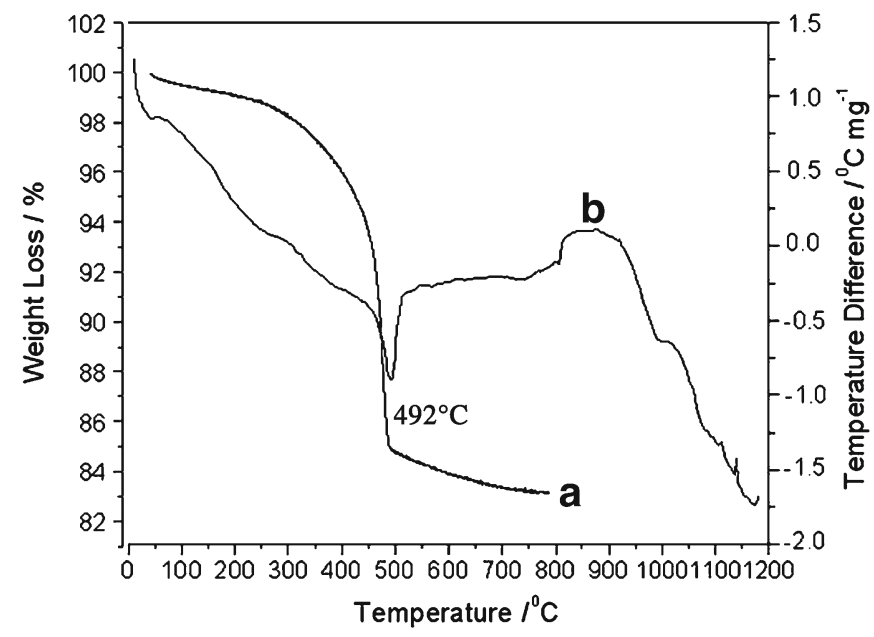

Figure 5. Thermal analysis of the precursor $\gamma-\mathrm{AlOOH}$ nanowires: a. TG curve and b. DTA curve.

Truitt 1997). The endothermic peak at $492^{\circ} \mathrm{C}$ in DTA curve is assigned to a phase transformation of precursor $\gamma-\mathrm{AlOOH}$ converting to $\gamma-\mathrm{Al}_{2} \mathrm{O}_{3}$.

\section{Conclusions}

In summary, single-crystalline $\gamma-\mathrm{Al}_{2} \mathrm{O}_{3}$ nanowires have been successfully synthesized by thermal decomposition of $\gamma$ $\mathrm{AlOOH}$ precursors which were prepared by the hydrothermal method using anhydrous $\mathrm{AlCl}_{3}, \mathrm{NaOH}$ and $\mathrm{NH}_{3}$ as the starting materials at $200^{\circ} \mathrm{C}$ for $24 \mathrm{~h}$. The $\gamma$-AlOOH nanowires have a diameter of $20-40 \mathrm{~nm}$, length above $600 \mathrm{~nm}$ and aspect ratio above 30 . The $\mathrm{pH}$ value and the mixed precipitation play important roles in the formation of the precursor $\gamma$ $\mathrm{AlOOH}$ nanowires. The size and morphology of $\gamma-\mathrm{Al}_{2} \mathrm{O}_{3}$ can be well preserved during the transformation from $\gamma$ $\mathrm{AlOOH}$ to $\gamma-\mathrm{Al}_{2} \mathrm{O}_{3}$.

\section{Acknowledgements}

This work was supported by the Shanghai Leading Academic Discipline Project (J51402). We thank Analysing Centre of Shanghai Jiaotong University for the characterization of TEM and XRD.

\section{References}

Baughman R H, Zakhidov A A and Hee W A 2002 Science 297787 Chen X Y and Lee S W 2007 Chem. Phys. Lett. 438279

Chen X Y, Huh H S and Lee S W 2007 Nanotechnology 18285608 Han W, Fan S and Li Q 1997 Science 2771287

Hellmig R J and Ferkel H 1999 Phys. Status Solidi (a) 175549 Hu J, Odom T W and Lieber C M 1999 Acc. Chem. Res. 32435 Iijima S 1991 Nature 35456

Jun Y W, Choi J S and Cheon J W 2006 Angew. Chem. Int. Ed. 45 3414 
KnoK zinger H and Ratnasamy P 1978 Catal. Rev.-Sci. Eng. 1731 Kong J, Soh H T and Cassell A M 1998 Nature 385878

Liu X and Truitt R E 1997 J. Am. Chem. Soc. 1199856

Ma M G, Zhu Y J and Xu Z L 2007 Mater. Lett. 611812

Moc M, Zhang L D and Yuan Z 1995 Nanostructured Mater. 595

Pan Z W, Dai Z R and Wang Z L 2001 Science 2911947

Peng H X, Fan Z, Mudher D S and Evans J R G 2002 Mater. Sci. Eng. A335 207
Rao C N R, Deepak F L, Gundiah G and Govindaraj A 2003 Prog. Solid State Chem. 315

Tang C C, Fan S S, Li P, Lamy de la Chapelle M and Dang H Y 2001 J. Cryst. Growth 224117

Wang X and Li Y D 2006 Inorg. Chem. 457522

Xia Y, Rogers J A and Paul K 1999 Chem. Rev. 991823

Zhou J, Deng S Z, Chen J, She J C and Xu N S 2002 Chem. Phys. Lett. 365505 